\title{
Single-Nucleotide Polymorphism of the FKBP5 Gene and Childhood Maltreatment as Predictors of Structural Changes in Brain Areas Involved in Emotional Processing in Depression
}

\author{
Leonardo Tozzi', Angela Carballedo',2, Friedrich Wetterling', Hazel McCarthy', Veronica O'Keane ${ }^{3}$, \\ Michael Gill ${ }^{4}$, Derrek Morris ${ }^{4}$, Ciara Fahey ${ }^{4}$, James Meaney ${ }^{2}$ and Thomas Frodl ${ }^{*, 1,2,5,6}$ \\ 'Integrated Neuroimaging Group, Department of Psychiatry,Institute of Neuroscience, School of Medicine, Trinity College Dublin, University of \\ Dublin, College Green, Dublin, Ireland; ' Centre of Advanced Medical Imaging, St James's Hospital, Trinity College Dublin, Dublin, Ireland; \\ ${ }^{3}$ Departement of Psychiatry and Adelaide and Meath Hospital Dublin, Dublin, Ireland; ${ }^{4}$ Neuropsychiatric Genetics Group, Department of \\ Psychiatry, Institute of Neuroscience, School of Medicine, Trinity College Dublin, University of Dublin, College Green, Dublin, Ireland; ${ }^{5}$ Department of \\ Psychiatry, University of Regensburg, Regensburg, Germany; ${ }^{6}$ Department of Psychiatry and Psychotherapy, Otto von Guericke University \\ Magdeburg, Magdeburg, Germany
}

The gene expressing the FK506 binding protein 51 (FKBP5) is involved in the regulation of glucocorticoid receptor sensitivity. The rs 1360780 SNP in this gene ( $T$ allele vs C homozygous) has been found to be associated with major depressive disorder (MDD). The aim of our study was to investigate whether this polymorphism might be associated with altered brain structure and function in a cohort of 40 patients with MDD and 43 healthy controls. A functional magnetic resonance imaging ( $\mathrm{M}$ RI) emotional attention task was employed. Diffusion tensor imaging (DTI) was also conducted, extracting mean diffusivity (MD) and fractional anisotropy (FA) from brain areas that showed functional differences between patients expressing the two alleles of the rs I 360780 SNP. Finally, the effect of the interaction of childhood adversity as measured by the Childhood trauma Questionnaire (CTQ) and rs I 360780 allele status was analyzed in relation to DTI measures using a general linear model. All results presented are family-wise error (FWE) corrected. Functional interactions were found between genotype and diagnosis $(p<0.0 \mathrm{l}$ ). Patients carrying the high-risk allele, compared with patients not carrying it, showed reduced activity in the rolandic operculum, Heschl gyrus, insula, parahippocampal gyrus, posterior cingulate cortex, inferior frontal gyrus ( $p<0.05$ for all measures); and increased MD and reduced FA measures in many of these regions $(p<0.05)$. An interaction between CTQ scores and allele status was associated with DTI changes in the insula, rolandic operculum, and inferior frontal gyrus. Here, the presence of both the high-risk allele and higher CTQ scores was associated with higher MD and lower FA values $(p<0.05)$. In conclusion, MDD patients expressing the T allele of rs 1360780 , compared with C homozygous patients, exhibit functional and structural differences in areas involved in emotional perception and inhibition. The interaction between the $T$ allele and childhood maltreatment explained our structural findings in these regions, suggesting that their altered maturation and function might be influenced by early chronic stress in the presence of this genetic trait.

Neuropsychopharmacology (2016) 4I, 487-497; doi:I0.1038/npp.20I5.170; published online 8 July 2015

\section{INTRODUCTION}

As an essential part of research on major depressive disorder (MDD), many studies have focused on finding associations between the disease and genetic factors. Ultimately, the goal is to gain a better insight into interactions that might help define subsets of patients at risk or more likely to respond to certain therapies. Examples of the genes that have

*Correspondence: Professor T Frodl, Department of Psychiatry and Psychotherapy, University of Regensburg, Medizinische Einrichtungen des Bezirks Oberpfalz-KU, Universitätsstraße 84, Regensburg 93053, Germany, Tel: +49 9419412017, Fax: +49 941 941 62017, E-mail: thomas.frodl@medbo.de

Received 2 March 2015; revised I May 20I5; accepted 20 May 20 I5; accepted article preview online 16 June 2015 been investigated in this regard include genes involved in monoaminergic signalling such as the monoamine oxidase A (MAOA) (Gizatullin et al, 2006; Bellivier et al, 1998), tryptophan hydroxylase 1 (TPH1) (Gizatullin et al, 2006), and serotonin transporter (5-HTT) genes (Bellivier et al, 1998; Caspi et al, 2003; Frodl et al, 2010). Mediators of neuronal plasticity have been studied as well, such as the brain derived neurotrophic factor (BDNF) (Arlt et al, 2013; Lavebratt $e t a l, 2010)$ and BicC family RNA binding protein 1 (BICC1) (Bermingham et al, 2012) genes. However, all genome-wide association analyses that have been performed have so far found inconclusive results regarding the association between SNPs in all these genes and MDD, suggesting that environmental factors may be crucial for developing the disease regardless of genetic vulnerability (Gyekis et al, 2013; 
Bosker et al, 2011; Clarke et al, 2010; Cohen-Woods et al, 2013).

Compatible with the hypothesis of altered stress systems and immune response dysfunction in MDD (Miller et al, 2009), recent data have shown an association between the disease and allelic variants of genes involved in glucocorticoid receptor (GR) regulation. The gene expressing the FK506 binding protein 51 (FKBP5), in particular, is involved in the regulation of GR sensitivity (Scharf et al, 2011). The overexpression of this protein can reduce hormone binding affinity and nuclear translocation of GR, downregulating the expression of anti-inflammatory proteins in neuronal nuclei (Wochnik et al, 2005).

Genetic variants in this gene have been found to be suggestively associated with MDD (Gillespie et al, 2009), although not always achieving full statistical significance (Lavebratt et al, 2010). Furthermore, a study has also found an independent and interactive involvement of FKBP5 (as well as GRIK4 and HTR2A) in antidepressant treatment response (Horstmann et al, 2010), emphasizing the potential importance for treatment in the study of this gene.

The rs1360780 single-nucleotide polymorphism (SNP) of the FKBP5 gene has been especially explored and its allele $\mathrm{T}$ has been reported as possibly associated with depression (Lavebratt et al, 2010; Gillespie et al, 2009). Further studies have revealed an association between this genotype and MDD in specific patient cohorts and the hypothesis has arisen that an increased risk of developing MDD in carriers of the $\mathrm{T}$ allele of rs 1360780 could be present only following its interaction with a significant amount of chronic stress, such as the one undergone by gastric cancer patients (Kang et al, 2012) or victims of childhood maltreatment (Gillespie et al, 2009). In the past few years, a significant association has also been reported between depression, FKBP5 allele $\mathrm{T}$ carrier status and an impaired regulation of the endocrine HPA axis (Menke et al, 2013). Additionally, allelic differences in this gene have been found to be associated with alterations in cingulum anatomy measured using diffusion tensor imaging (DTI) in patients suffering from post-traumatic stress disorder (PTSD), suggesting that it may have a role in determining white-matter integrity and increased vulnerability for psychiatric disorders (Fani et al, 2014).

To better understand the way SNPs of FKBP5 may affect brain function, some studies have investigated whether carriers of the high-risk $\mathrm{T}$ allele of rs1360780 responded differently to well-established functional magnetic resonance imaging (fMRI) paradigms. In healthy participants, the $\mathrm{T}$ allele of rs1360780 has been thus associated with an increase in BOLD responses in the hippocampus during a dot probe task, accompanied by alterations in hippocampal shape and with an attention bias toward threat (Fani et al, 2013; Holz et al, 2014). Another study has also found, in participants carrying the $\mathrm{T}$ allele, an association between increased activity in the dorsal amygdala during a face-recognition paradigm and self-reported childhood emotional neglect (White et al, 2012). Regarding depressed patients, on the other hand, the FKBP5 gene and childhood adversity have been shown to interact and to be associated with abnormal activity in the amygdala, hippocampus, and orbitofrontal cortex (Holz et al, 2014).

All these findings support the hypothesis that there might be a neurobiological interplay between variants of the FKBP5 gene, stressful environmental factors (such as childhood adversity) and MDD, leading to specific changes in brain anatomy and function. Furthermore, during tasks eliciting emotional responses in patients, specific patterns may arise that differ between the genetically defined sub-samples.

The aim of our study was to investigate the differences in brain function and anatomy between patients affected by MDD and healthy controls (HCs) in relation to the allelic variants of the rs1360780 SNP of the FKBP5 gene. Furthermore, we wanted to investigate whether these differences might be explained by the interaction between the presence of the high-risk $\mathrm{T}$ allele of rs1360780 and environmental stress (in particular childhood adversity).

To achieve our goal, we employed MRI during a task centred around attentional focus on emotional picture stimuli. By studying brain activity following the emotional evaluation of stimuli and those following their cognitive processing, we wanted to gain insight into the extent of emotional inhibition following the exposure to the stimulus: an ability that has been found impaired in depressed patients (Goeleven et al, 2006). We then located areas presenting differences in activity between patients carrying the high-risk $\mathrm{T}$ allele and those being homozygous for the $\mathrm{C}$ allele. Next, we used DTI to assess whether these regions also presented morphological changes between these same two subgroups. Finally, we have focused on the hypothesis that the genetic factor alone would not be sufficient to explain our morphological findings in depressed patients, but that its interaction with early life stress would highlight its contribution. Therefore, we have investigated the effects of the interaction of childhood adversity and rs 1360780 allele status in explaining the DTI measures obtained by the use of a general linear model.

\section{MATERIALS AND METHODS}

\section{Sample}

The study included 40 adult patients (13 males) with MDD from the mental health services of the Tallaght Hospital, Dublin or St James's Hospital, Dublin. The diagnosis of these patients was based on DSM-IV criteria, made by the responsible consultant and confirmed by an independent psychiatrist using the SCID interview. Fortythree HC subjects (20 males) from the local community were also recruited.

Exclusion criteria were age $<18$ or $>65$, history of neurological or comorbid psychiatric disorders (Axis I or Axis II), other severe medical illness, head injury, or substance abuse. Demographic variables, inclusion and exclusion criteria were documented using a standardized questionnaire and through a structured interview (SCID-I) by a psychiatrist.

Written informed consent was obtained from all participants after being given detailed description of the study, which was designed and performed in accordance to the ethical standards laid out by the Declaration of Helsinki and was approved by the ethics committee of St James and Tallaght hospitals, Dublin. 


\section{Rating Instruments}

Self and observer rated scales were also filled out for all participants included in the study. The rating scales that were used comprised: the Hamilton Rating Scale for Depression (Hamilton, 1986) and Beck's Depression Inventory (BDI-II) (Beck et al, 1961). The 28-item short form of the Childhood Trauma Questionnaire Short Form (CTQ-SF) was used to assess adversity during childhood and teenage years (Bernstein and Fink, 1998). The sum of its five sub-items (emotional, physical and sexual abuse, emotional, and physical neglect) was calculated and used as a continuous variable to evaluate the severity of childhood maltreatment for each participant.

\section{Genetic Analysis}

rs1360780 was genotyped from blood in this sample using a Taqman SNP Genotyping Assay on a 7900HT Sequence Detection System (Applied Biosystems). The call rate for the Taqman genotyping was $>95 \%$ and all samples were in Hardy-Weinberg equilibrium $(p>0.05)$. Along with the test samples, a number of HapMap CEU DNA sample positive controls (www.hapmap.org) and non-template negative controls were genotyped for quality control purposes. For positive controls, all genotypes were found to be concordant with available online HapMapdata. All non-template samples returned a negative result. rs1360780 is in Hardy-Weinberg equilibrium $(p>0.05)$ in this sample. Our test SNP at FKBP5 has a minor allele frequency (MAF) of 0.42 according to the University of California Santa Cruz Genome Browser. T is the minor allele and because homozygous TT samples are rare in our sample, we grouped them with heterozygous TC samples for analysis.

\section{MRI Data Acquisition}

Magnetic resonance images were obtained with a Philips Achieva 3 Tesla MRI scanner. The protocol consisted of the acquisition of a high-resolution 3D T1-weighted structural data set (SPGR sequence with TR/TE $=8.5 / 3.9 \mathrm{~ms}$, field of view (FOV) of FH (foot to head): $256 \mathrm{~mm}$, AP (anterior to posterior): $256 \mathrm{~mm}$, RL (right to left): $160 \mathrm{~mm}$; and a matrix of $256 \times 256$ and $1 \mathrm{~mm} 3=$ spatial resolution), followed by an fMRI experiment (SE-EPI sequence with TR/TE $=2000 / 35$ $\mathrm{ms}$, in place resolution $=3 \times 3 \mathrm{~mm}^{2}, 4.8 \mathrm{~mm}$ slice thickness, 550 dynamic scans each with $2 \mathrm{~s}$ duration). A DTI scan was then performed based on high angular resolution diffusion imaging (HARDI) with 61 diffusion directions (field of view (FOV): $200 \times 257 \times 126 \mathrm{~mm}, 60$ slices, no gap, spatial resolution: $1.8 \times 1.8 \times 2.1 \mathrm{~mm}, \mathrm{TR} / \mathrm{TE}=12561 / 59 \mathrm{~ms}$, flip angle $=90^{\circ}$, half k-space acquisition was used (half scan factor $=0.68$ ), SENSE parallel imaging factor $=2.5, b$ values $=$ $0,1200 \mathrm{~s} / \mathrm{mm}$, with SPIR fat suppression and dynamic stabilization in an image acquisition time of $15 \mathrm{~min} 42 \mathrm{~s}$ ).

\section{fMRI Task}

An attentional cognitive emotional task was used in the fMRI experiment where participants were asked to process visual stimuli. The task, fMRI pre-processing, and primary data analysis were described in detail in Lisiecka et al (2011). The task was event related and consisted of 180 pseudorandomized trials. Each trial in the task was $4 \mathrm{~s}$ long and consisted of a fixation cross, followed by a viewing stage in which positive, negative, or neutral rectangular pictures from the International Affective Picture System database (IAPS) were shown. After seeing the picture, participants were either asked to focus on the emotion elicited by the picture and answer whether this was positive, negative, or neutral or had to answer a question about its orientation (horizontal or vertical). The same amount (Sprengelmeyer et al, 2011) of geometrical and emotional trials was delivered for each of the three valences (positive, negative, and neutral).

As behavioral measures, the response times and the accuracy of responses by indicating the number of 'incorrect' guesses was compared for each emotional condition between patients and controls with $T$-tests and an ANOVA correcting for age and sex.

\section{fMRI Analysis}

Pre-processing steps included realignment to correct for motion. Participants were excluded when movement parameters exceeded $3 \mathrm{~mm}$. Then co-registration of each participant's structural image to the mean of the motion corrected functional images, slice time correction, spatial normalization, and smoothing using an $8 \mathrm{~mm}$ full width, half maximum (FWHM) Gaussian kernel were applied. Data were analyzed with Statistical Parametric Mapping 12 (SPM12, http://www.fil.ion.ucl.ac.uk/spm). Motion correction values were added as a covariate. In first-level analyses, $t$-test contrasts were calculated comparing emotional (judging the emotional content) and geometrical trials of each valence $v s$ a baseline condition (fixation cross).

A $2 \times 2 \times 2 \times 3$ full-factorial model was performed with SPM12 on the resulting contrasts where the first factor was diagnosis group (MDD or $\mathrm{HC}$ ), the second factor was the presence of the $\mathrm{T}$ allele in the rs1360780 SNP of the FKBP5 gene ( $\mathrm{T}$-allele carrier or $\mathrm{C}$ homozygous), the third factor was the processing of the stimulus (emotional or geometrical), and the fourth factor was its emotional valence (positive, negative, or neutral), while age, gender, and medication (entered as medication type: 0 for unmedicated patients and controls, 1 for SSRIs, and 2 for dual acting antidepressants) were used as covariates. A whole brain family-wise error (FWE) correction with $p<0.05 \quad(p<0.01$ for interaction testing) was performed in all comparisons to ensure statistical significance of our findings. The xjView toolbox (http://www.alivelearn.net/xjview) was used to localize the brain areas in which the significant differences were located in a standard stereotactic space (template from the Montreal Neurological Institute (MNI)).

\section{DTI Analysis}

Data were pre-processed using Explore DTI (http://www. exploredti.com). Diffusion tensor estimation was conducted with a robust non-linear approach; motion correction was applied to all data using a cubic interpolation and restore function; eddy current correction was used, as well as EPI correction based on the T1 images of each subject; we reviewed the DTI data by visually inspecting the slice images. Head movement during scanning was less than $3 \mathrm{~mm}$ in $\mathrm{x}, \mathrm{y}$, 
Table I Demographics of Our Sample

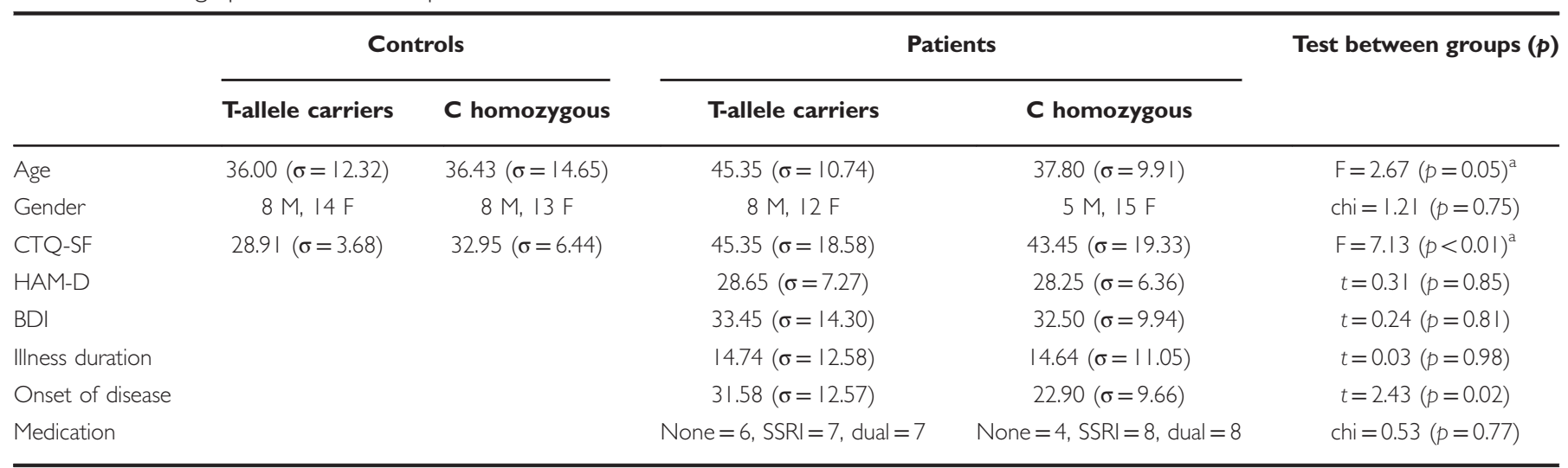

${ }^{\text {aP }}$ ost hoc $t$-tests between groups were not significant for age $(p>0.05)$. CTQ scores were higher among patients compared with controls ( $p$ ost hoc $t$-test $p<0.0$ I). CTQSF, childhood trauma questionnaire, short form; HAM-D, Hamilton rating scale for depression; BDI, Beck depression inventory; M, male; F, female; SSRI, selective

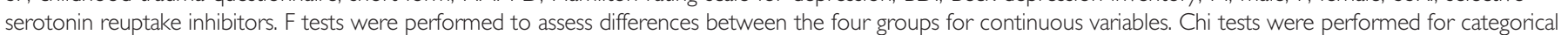
variables. T-allele carriers $=$ carriers of the T allele of rs I 360780. CC homozygous $=\mathrm{C}$ homozygous for the C allele of rs $\mathbf{3 6 0 7 8 0 . ~ S t a n d a r d ~ d e v i a t i o n s ~ a n d ~} p$-values are given.

$\mathrm{z}$ directions. After pre-processing, we used the 'extract diffusion measures from atlas labels' tool in ExploreDTI, which warps each subject's image into standard space and defines masks for it based on the aal atlas (http://www. cyceron.fr/web/aal_anatomical_automatic_labeling.html).

These masks are then warped back into subject space and used for the extraction of DTI measures from the original DTI images for each subject.

In particular, we compared mean diffusivity (MD) and fractional anisotropy (FA) values of patients with the T allele of rs1360780 with those of patients homozygous for the $\mathrm{C}$ allele bilaterally in the anatomical areas where we found significant differences between the same groups during the fMRI task: insula and neighboring rolandic operculum; Heschl gyrus; superior temporal lobe; parahippocampal gyrus; posterior cingulate cortex; inferior frontal gyrus, pars triangularis.

\section{Interaction Modelling}

We defined and tested separate general linear models as implemented in SPSS Statistics version 22 (IBM) using each of our significant DTI findings as a dependent variable and, as an independent variable, the interaction between childhood maltreatment (CTQ scores) and the presence of the $\mathrm{T}$ allele of rs1360780. In the model, age, sex, and medication were also included as confounders. Statistics were considered to be significant when $p<0.0125$ considering testing for four different regions that were found to be significant in the fMRI and DTI analysis described above.

\section{RESULTS}

\section{Sample}

In all, 20 of the depressed patients and 22 of the HCs were found to be carriers of the $\mathrm{T}$ allele of rs1360780. There was no significant difference overall between participant groups regarding gender and age. CTQ scores were higher in patients compared with controls.
Among MDD patients, there were no significant differences between participants carrying the $\mathrm{T}$ allele and $\mathrm{CC}$ participants regarding HAM-D scores, BDI scores, illness duration, gender, childhood maltreatment, and medication. $\mathrm{T}$-allele carriers had a later onset of disease compared with $\mathrm{C}$ homozygous patients. For a summary of demographics and clinical variables, see Table 1 .

\section{Behavioral}

Patients with MDD made significantly more mistakes than HCs in the evaluation of geometrical trials involving neutral stimuli (mean of errors: patients $=4.11$, controls $=2.40$; $t=-2.43, p=0.02)$, regardless of age and sex $(\mathrm{F}=6.79$, $p=0.01)$. Higher response times for depressed patients were observed across all trials and appeared to be due to both diagnosis and age $(p<0.01)$. No behavioral differences were found between patients expressing the $\mathrm{T}$ allele of rs 1360780 and $\mathrm{C}$ homozygous patients (all tests $p>0.05$ ).

\section{fMRI}

For a summary of our fMRI results, see Table 2. For the mean task activation across all stimuli and across all participants, see (Figure 1, Supplementary Material). Interactions between the type of trial or valence and the allele factor and diagnosis were not significant. We found a significant interaction between genotype and diagnosis in the following brain areas: left and right superior parietal lobules; right frontal superior orbital gyrus; right frontal middle orbital gyrus; left middle occipital gyrus; left frontal inferior orbital gyrus; left insula and left superior temporal lobe (Supplementary Figures 2 and 3, Supplementary Material).

C homozygous controls showed, compared with $\mathrm{C}$ homozygous patients, an increased response in the right middle frontal gyrus, in the left inferior frontal gyrus (pars triangularis) and in the left middle frontal gyrus, regardless of trial and valence. Conversely, controls carrying the $\mathrm{T}$ allele compared to patients with the same genotype showed a greater 
Table 2 PMRI Findings

\begin{tabular}{llllllll}
\hline Contrast & $k$ & $T$ & & \multicolumn{3}{c}{ Peak coordinates } & Region \\
\cline { 3 - 5 } & & & $x$ & $y$ & $z$
\end{tabular}

Controls $>$ patients

$\begin{array}{lrr}116 & 6.05 & 5.93 \\ 107 & 5.58 & 5.49 \\ & 5.46 & 5.37 \\ & 4.9 & 4.84 \\ 12 & 5.22 & 5.15 \\ 12 & 4.81 & 4.75 \\ & 4.66 & 4.6\end{array}$

$C C>T$ carriers

$\begin{array}{rrrr}629 & 6.2 & 6.07 & \\ & 5.74 & 5.64 & 2 \\ & 5.72 & 5.62 & 33 \\ 157 & 5.87 & 5.76 & -36 \\ & 5.37 & 5.76 & -3 \\ & 4.92 & 4.85 & -3 \\ 141 & 7.22 & 7.03 & -2 \\ 45 & 5.73 & 5.63 & - \\ 36 & 5.39 & 5.31 & -2 \\ 13 & 4.96 & 4.89 & \end{array}$

Interaction: FKBP5*diagnosis ( $p<0.01$ FWE)

$$
1544
$$$$
51
$$$$
F=66.25
$$$$
F=52.05
$$$$
7.77
$$$$
F=50.33
$$$$
6.81
$$$$
F=65.07
$$$$
6.81
$$

51

$$
\begin{aligned}
& F=41.56 \\
& 48 \quad F=39.05
\end{aligned}
$$$$
F=32.92
$$$$
F=35.81
$$$$
F=31.82
$$$$
F=29.31
$$

CC controls > CC patients

$\begin{array}{rrr}338 & 5.79 & 5.69 \\ 160 & 4.9 & 4.84 \\ 45 & 5.89 & 5.78 \\ & 4.84 & 4.78\end{array}$

$$
\begin{array}{r}
-24 \\
-45 \\
18 \\
18 \\
33 \\
-42 \\
-36 \\
-48 \\
-39 \\
-45
\end{array}
$$$$
-55
$$$$
-67
$$

41
-37
-49
-58
-55
35
35

Region

$R$ middle frontal gyrus
$R$ hippocampus
$R$ precuneus
$L$ lingual gyrus
$L$ lingual gyrus
$R$ middle frontal gyrus
$R$ middle frontal gyrus

$\mathrm{R}$ anterior cingulate cortex

$\mathrm{R}$ middle frontal gyrus

$R$ middle frontal gyrus

$L$ middle frontal gyrus

$L$ inferior frontal orbital gyrus

$L$ insula

$L$ hippocampus

$L$ posterior cingulate cortex

$L$ frontal superior orbital gyrus

$R$ frontal medial orbital gyrus

$-13$

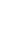

superior parietal lobule

L superior parietal lobule

$R$ superior parietal lobule

$R$ frontal superior orbital gyrus

$R$ frontal middle orbital gyrus

$L$ middle occipital gyrus

$L$ frontal inferior orbital gyrus

$L$ superior temporal lobe

$L$ insula

$L$ superior temporal lobe
$R$ middle frontal gyrus
$L$ inferior frontal gyrus pars triangularis
$L$ middle frontal gyrus
$L$ middle frontal gyrus

$T$ carrier controls $>T$ carrier patients

192

6.39
5.96
5.26
5.28
5.26

CC patients $>T$ carrier patients (emotional)

$\begin{array}{ll}50 & 5.72 \\ 49 & 5.44 \\ 36 & 5.6 \\ 26 & 5.37\end{array}$

CC patients > T carrier patients (geometrical)

$$
27
$$

6.25
5.85
5.18
5.2
5.19

27
15
18
-24
-36

$$
-40
$$$$
-46
$$$$
-61
$$$$
-61
$$$$
-55
$$

51

2
-46
-31
-58

$-58$

17
R hippocampus

$\mathrm{R}$ precuneus

$R$ lingual gyrus

L superior parietal lobule

$\mathrm{L}$ inferior parietal lobule 
Table 2 Continued

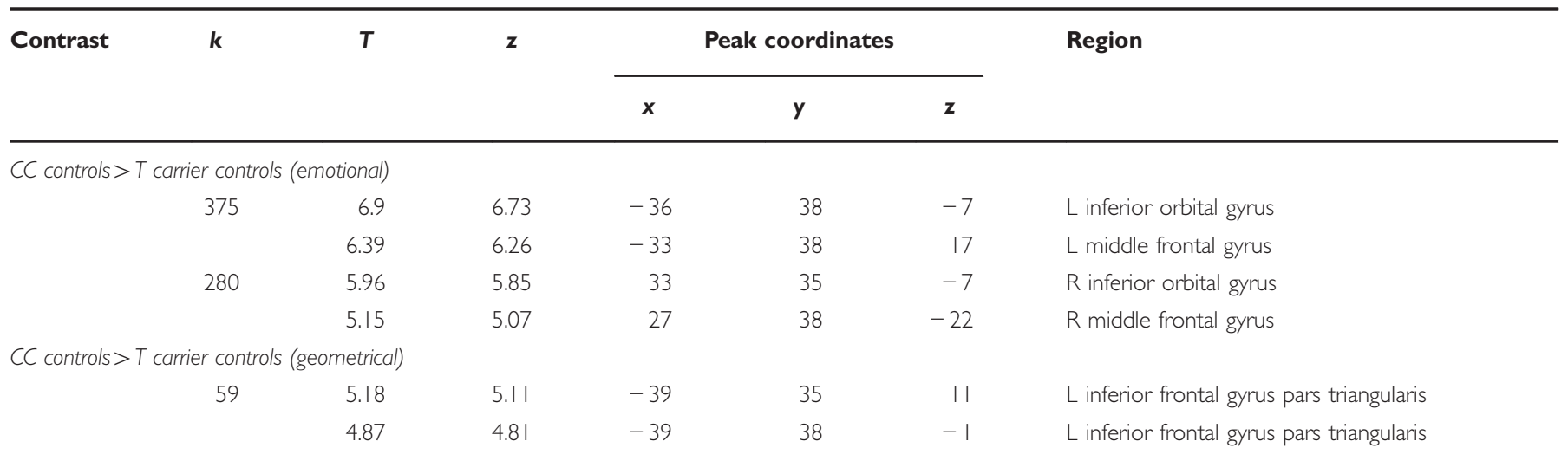

Contrasts between groups are given, all $p$-values are $<0.05$ whole brain family-wise error corrected unless otherwise stated. $k=$ cluster size (voxels). For each peak voxel: $T=$ value of T-test; $Z=z$-converted values of $T ; x, y, z=$ coordinates in $\mathrm{mm}$. T-allele carriers $=$ carriers of the $T$ allele of $r s \mid 360780$. CC homozygous $=C$ homozygous for the C allele of rs 1360780. L, left; R, right. Region of the peak was determined according to the aal atlas (http://wmw.cyceron.fr/web/aal__anatomical_ automatic_labeling.html).

activation in the right hippocampus, right precuneus, right lingual gyrus, and left superior and inferior parietal lobules.

In response to emotional trials, $\mathrm{C}$ homozygous patients showed an increased response compared with patients carrying the $\mathrm{T}$ allele in the following regions: left superior temporal lobe and insula; left parahippocampal gyrus; left posterior cingulate cortex, precuneus, and lingual gyrus. While judging the orientation of pictures, regardless of valence, they showed an increased response compared with patients carrying the $\mathrm{T}$ allele in the right inferior frontal gyrus, pars triangularis (Figure 1).

During emotional trials, $C$ homozygous controls had an increased response compared with controls carrying the $\mathrm{T}$ allele in the left and right inferior orbital gyri and in the left and right middle frontal gyri. Similarly to patients, after geometrical trials, they showed an increased response compared with controls carrying the $\mathrm{T}$ allele in the left inferior frontal gyrus, pars triangularis.

To test whether differences in the behavioral results could provide any further information to our model, we ran two additional second level analyses. The first one included the amount of errors in judging geometrical neutral pictures for each subject (the only trial where a significant behavioral difference was found) as an additional covariate. In the second, we included as a covariate the average reaction time for every subject, since differences were evident overall across trials. The addition of these covariates did not change the results in any way, leading us to believe that indeed diagnosis and age (already included in the model) were sufficient in explaining the behavioral differences in errors and reaction times, confirming the result of our previous ANOVA. Also, childhood trauma was significantly more present in the depressed patients group, raising the question whether group differences might not be attributable to the diagnosis itself, but to the environmental risk factor childhood maltreatment. Therefore, we repeated the same procedure with a third second level model where CTQ scores were added as a covariate for each participant. Again, no differences were found in the results.

\section{DTI}

For a summary of our DTI results, see Table 3 . Since activity in the inferior frontal gyrus pars triangularis was found to differ during cognitive trials between $\mathrm{C}$ homozygous participants and T-allele carriers also in controls, we decided to study the DTI measures in this region in controls as well, but we found no significant difference $(p=0.15)$.

Increased $\mathrm{MD}$ values were found in patients carrying the $\mathrm{T}$ allele of rs1360780 compared with homozygous $\mathrm{C}$ patients in the left inferior frontal gyrus pars triangularis, left and right rolandic operculum, left and right insula, left and right Heschl gyrus. Regarding FA values, we found them decreased in the left rolandic operculum and in the left insula. Comparisons between the two groups in the other regions of interest were not significant.

\section{Interaction Modelling}

For a summary of our significant models fit and factors, see Table 4.

Dependent variables used in our models were normally distributed (Kolmogorov-Smirnov test $p>0.05$ ). Diagnostic procedures were run on all linear models to ensure a good fit of the data, including standardized and non-standardized residual plotting and marginal model plots. Our model yielded a successful and valid fit, with the interaction between rs1360780 allele status and CTQ scores being the only significant predictor, for the left and right insula $\mathrm{MD}$, the left rolandic operculum $\mathrm{MD}$ and $\mathrm{FA}$ and for the left frontal inferior gyrus, pars triangularis, MD. After correcting for multiple comparisons, the interaction was still significant for the left rolandic operculum, both for $\mathrm{MD}$ and for FA (Figure 2).

These models did not achieve a significant fit if the main effects of CTQ scores and rs1360780 allele status were entered as separate, non-interacting variables. In all other regions of interest, the interaction between CTQ scores and rs1360780 allele status did not show any significant role in predicting DTI measurements. 
a
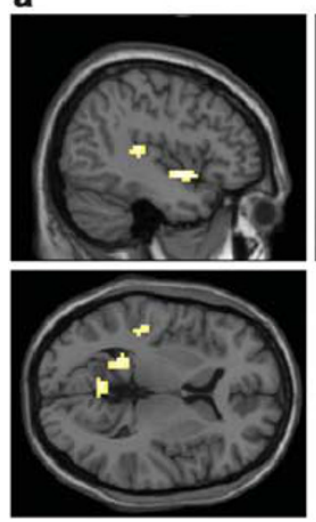

b
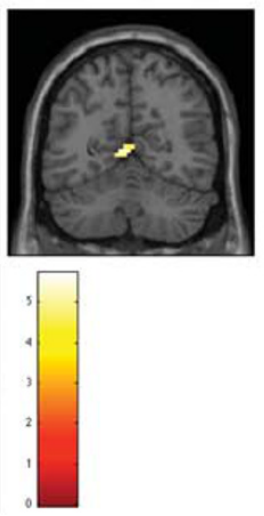
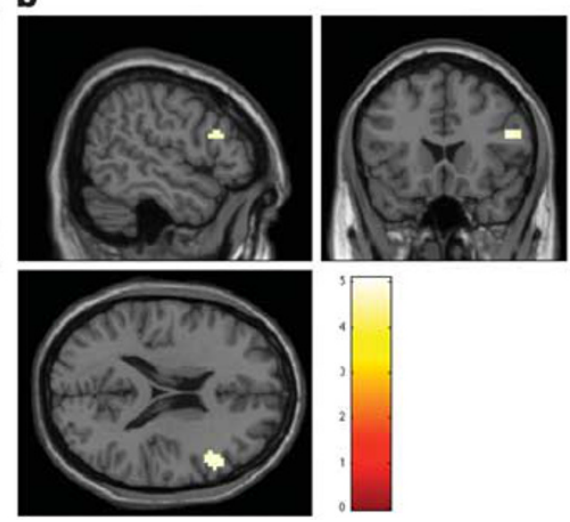

Figure I Functional results of the contrast between C homozygous patients and patients carrying the T allele of rs I 360780 . Areas of differential activation for emotional (a) and geometrical (b) trials are shown in standard MNI space in axial, coronal and sagittal views. Colour scales represent the $t$-values of the contrasts ( $p<0.05$ whole brain FWE corrected, cluster size threshold: 10 voxels).
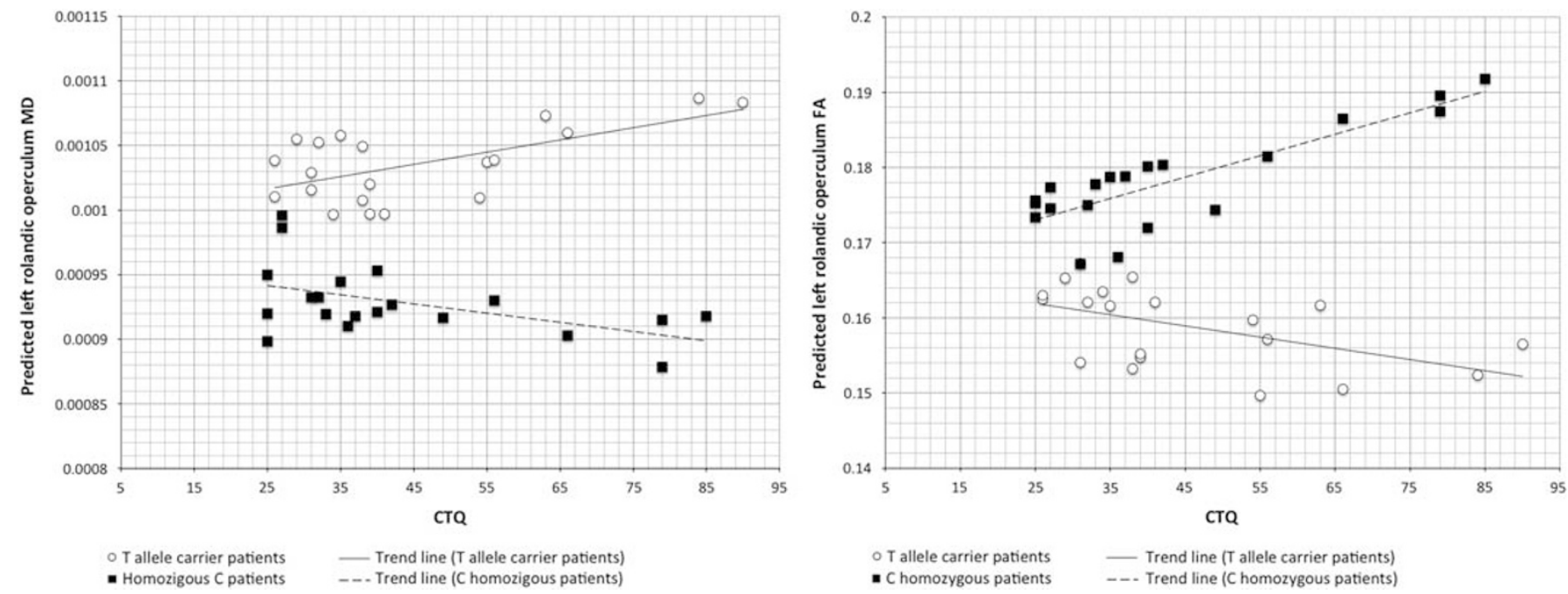

Figure 2 Mean diffusivity (MD) and fractional anisotropy (FA) values in the left rolandic operculum in depressed patients as predicted by the Childhood Trauma Questionnaire (CTQ) in our general linear model. CTQ has proven to be the only significant predictor of MD and FA values in this region, also surviving multiple comparisons testing. Its effect shows an interaction with the genotype of patients, with the difference between the two genotypes becoming more apparent the higher the CTQ score of the patient. MD is expressed in $10^{-3} \mathrm{~mm}^{2} / \mathrm{s}$.

None of the independent variables included in our models were successful at explaining the functional responses measured in any of the clusters. We have also tested with an analogous general linear model the possible role of CTQ scores in predicting amygdalar responses to negative emotional stimuli, as reported from the previous literature in HCs (Dannlowski et al, 2012). However, we do not report any significant fit both for negative cognitive and for emotional trials $(p>0.5)$.

\section{DISCUSSION}

Our task has been successful in discriminating between patients with MDD and HCs by showing a decreased response to emotional stimuli for patients in several areas that have already been extensively reported in the literature, such as the hippocampus and temporal lobe, the prefrontal cortex and the posterior cingulate cortex/precuneus (see Fitzgerald et al, 2008).
The hippocampus, temporal lobe, prefrontal cortex, and posterior cingulate cortex seem to be overall hypoactive in response to emotional stimuli in all our participants carrying the $\mathrm{T}$ allele of rs1360780, suggesting that this gene may indeed have an impact on areas relevant for the disease even in HCs. Animal studies found the FKBP5 gene to be especially expressed in some of these regions, such as the hippocampus (Scharf et al, 2011) and recent studies have shown the impact of rs1360780 polymorphism on both anatomy and function of this area in healthy subjects (Fani et al, 2014; Fani et al, 2013).

Moreover, a significant interaction between diagnosis and allele status, mainly located in the superior temporal and parietal lobes, in the orbitofrontal gyri and in the insula was found. The functional differences in these areas could be exacerbated in the presence of both the disease and the $\mathrm{T}$ allele of rs1360780. Regarding the insula and superior temporal lobe, these regions have been found in MDD patients to consistently show a decreased activity during 
Table 3 DTI Findings

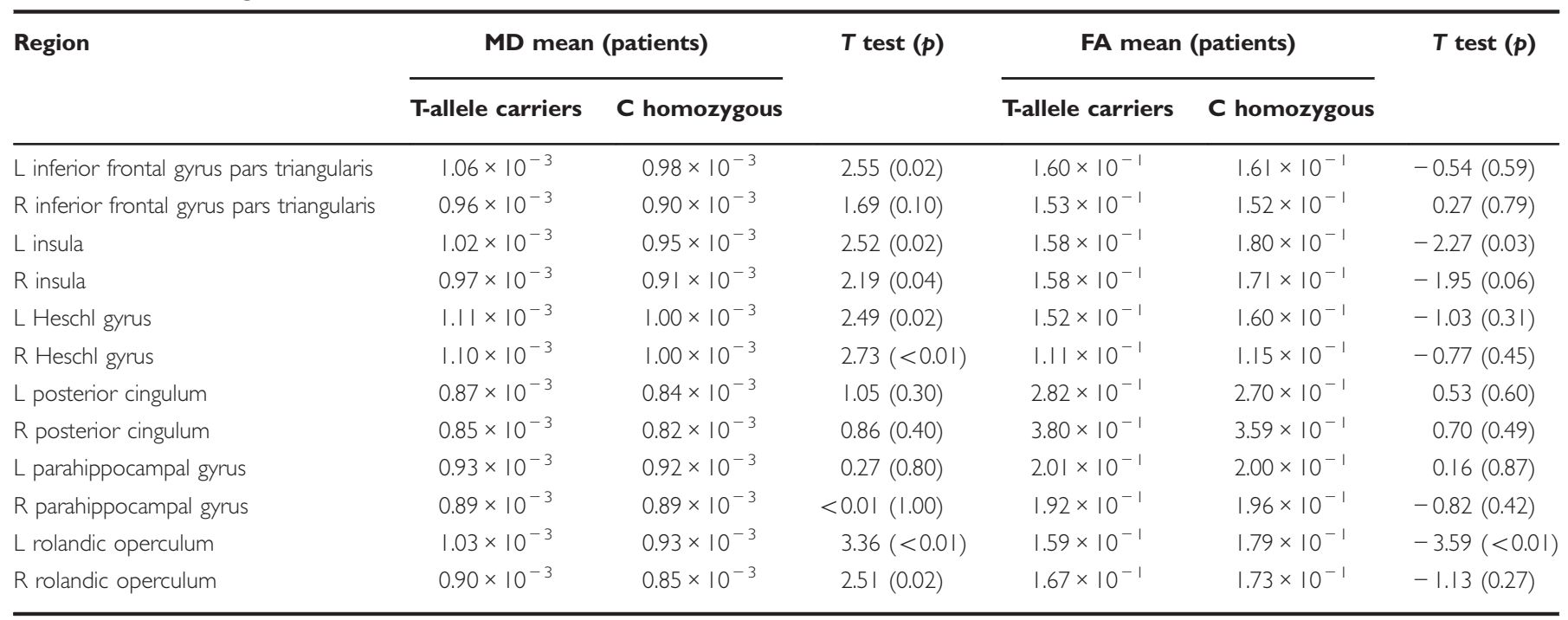

The results of $t$-tests for the contrasts between $C$ homozygous patients and T-allele carrier patients for rs 1360780 are given, in each region that we tested. MD is expressed in $10^{-3} \mathrm{~mm}^{2} / \mathrm{s}$. L, left; R, right.

Table 4 Results of Our General Linear Models in Depressed Patients

\begin{tabular}{lccccc}
\hline F tests $(\boldsymbol{p})$ & $\mathbf{L}$ insula MD & $\mathbf{R}$ insula MD & L rolandic operculum MD & L rolandic operculum FA & Left frontal inferior gyrus MD \\
\hline Corrected model & $3.04(0.02)$ & $2.84(0.02)$ & $3.12(0.02)$ & $2.69(0.03)$ & $2.37(0.05)$ \\
rsl360780*CTQ-SF & $3.58(0.04)$ & $4.51(0.02)$ & $5.74(<0.01)^{\mathrm{a}}$ & $5.92(<0.01)^{\mathrm{a}}$ & $3.12(0.05)$ \\
Sex & $0.85(0.36)$ & $0.01(0.92)$ & $0.17(0.69)$ & $0.20(0.66)$ & $<0.01(1)$ \\
Medication & $0.61(0.55)$ & $0.26(0.77)$ & $0.83(0.44)$ & $0.89(0.42)$ & $0.01(0.99)$ \\
Age & $3.01(0.09)$ & $2.70(0.11)$ & $1.38(0.25)$ & $0.01(0.92)$ & $3.33(0.77)$ \\
\hline
\end{tabular}

For each region in which we found significant results, the F values and $p$ are given for the fit of the overall model and of each factor. $r$ S $360780 * C T Q-S F=$ interaction between allele status and childhood trauma questionnaire scores. L, left; $R$, right.

asignificant findings after correction for multiple comparisons $(p<0.0125)$.

resting state studies, a relative lack of activation during induction of negative affect and an increase in activation with SSRI treatment (Fitzgerald et al, 2008). Furthermore, the volume of the insular cortex has been found to be negatively correlated to clinical symptoms in a sample of MDD patients (Sprengelmeyer et al, 2011).

In our study, patients carrying the high-risk $\mathrm{T}$ allele demonstrated significantly reduced activity in the insula following emotional stimuli than CC homozygous patients. The same was not observed for controls, suggesting that the effect of rs1360780 on the insula's function could become apparent when additional factors come into account that are related to the disease. One such factor could be childhood maltreatment that was significantly more pronounced in our patients with MDD compared with controls. This finding is consistent with the fact that the insula appears to deactivate in response to the acute activation of the stress hormone axis (Pruessner et al, 2008), such as the presentation of emotionally stressful material in our task. Genetic regulation of GR function together with the imbalances of the HPA axis characteristic of depression could explain how insular activity differs between subsets of patients.
Controls, on the other hand, exhibited differences in the middle and inferior frontal and orbitofrontal gyri that mimic more closely our findings from the overall contrast between allelic groups regardless of diagnosis. The same could be said for the reduced activation in the inferior frontal gyrus pars triangularis that we observed in both patients and controls carrying the $\mathrm{T}$ allele of rs 1360780 , especially during geometrical trials. This area is indeed specifically involved in cognitively challenging tasks, such as our geometrical trials, and its function has been found to be impaired in depressed patients (Harvey et al, 2005).

There have been many reports of an altered function in the amygdala in MDD during emotional processing (Sheline et al, 2001; Siegle et al, 2007; Victor et al, 2010; Suslow et al, 2010; Stuhrmann et al, 2011) but we did not see any effect in this region in our analysis. This could be due to a number of reasons. For example, we have considered brain activity in response to a task where the patient was asked to assess emotional content or geometrical shape of a picture. Therefore, the focus of our present analysis was on the cognitive processing following the question and being attention and emotional inhibition core components of this task, we were mainly expecting an involvement of cortical 
regions. Also, most studies reporting abnormal amygdalar activation in depression and investigating their links with other factors (such as childhood trauma) have used tailored tasks designed to elicit strong responses in this area, such as tasks involving emotionally salient faces (Sheline et al, 2001; Siegle et al, 2007; Victor et al, 2010; Suslow et al, 2010; Stuhrmann et al, 2011; Dannlowski et al, 2007, 2012). It is possible that our study design, which was focused on an exploratory investigation of the interaction between disease, brain function, structure, and a high-risk genotype, did not have enough power to investigate such fine-tuned regulation processes.

DTI demonstrated that MD values in the insula and neighboring temporal regions are significantly different between patients carrying the $\mathrm{T}$ allele of rs1360780 and those homozygous for the $\mathrm{C}$ allele. FA results, on the other hand, achieved significance in the left rolandic operculum and insula, confirming a different fibre orientation pattern in these areas.

Increased $\mathrm{MD}$ and reduced $\mathrm{FA}$ have been found to be associated with axonal degeneration, demyelination, decreased axonal density, and incomplete white-matter maturation (Alexander et al, 2011; Feldman et al, 2010). In $\mathrm{HCs}$, the $\mathrm{T}$ allele of rs 1360780 has been found to be associated with white-matter abnormalities (Fani et al, 2014) and changes such as these have been reported in patients with MDD as well (Frodl et al, 2012; Murphy and Frodl, 2011; Ugwu et al, 2014). Specifically, two studies (Abe et al, 2010; Shimony et al, 2009) reported higher MD and lower FA scores in the prefrontal cortex. These findings suggest that the high-risk $\mathrm{T}$ allele of rs1360780 (and subsequent modifications in stress hormone axis function) might have an impact on the maturation of these areas. Structural changes, in turn, might overlap with those associated with clinical depression and therefore become more evident and be related to the altered function we have found in the same regions.

Finally, we would like to point out how, in our patient group, the interaction between childhood abuse and genotype of rs1360780 successfully explained MD scores in the left insula, in the left and right rolandic operculum, and in the left inferior frontal gyrus, as well as FA in the left rolandic operculum. After correction for multiple comparisons, our finding was still significant in the left rolandic operculum, both for MD and for FA. This finding is consistent with the previous literature on the role of the allelic variants of FKBP5: a stressful environment achieves phenotypical relevance toward the modification of the stress hormone axis only in combination with the allelic variant, with childhood maltreatment having an especially significant impact (Binder et al, 2008; Ising et al, 2008).

This study presents some noteworthy limitations. First of all, due to the rarity of the $\mathrm{T}$ allele, we were forced to group homozygous and heterozygous patients together to achieve a sufficient group numerosity. Sample size overall is still small for a genetics study in each of the groups, but our choice of this SNP was guided by the previous consistent literature documenting findings in large samples. Also, it is still unclear how the gene might influence brain activity in the reported regions. Since the GR receptor is ubiquitously expressed, the relationship between the brain areas involved in emotional regulation and the T allele of FKBP5 is likely to be extremely complex and still needs to be elucidated. Further studies are therefore needed to confirm our findings in a larger sample and in respect to possible biomolecular mechanisms of rs1360780 action.

Also, we cannot possibly make any claims about the risk of developing MDD associated with carrying the $\mathrm{T}$ allele of the FKBP5 gene, being our study not longitudinal in nature. Additionally, we could not take many other factors into consideration that might have been associated with the condition, such as family history or environmental variables besides childhood maltreatment. Finally, many of our MDD patients were medicated and, although no significant difference in medication was found between the two genetically defined subgroups of patients and medication was used as a covariate in our functional analysis, we cannot completely exclude that some of the differences we reported between patients and controls might be due to this factor.

In summary, we show for the first time that allelic variants in the rs1360780 region of the FKBP5 gene are associated with differences in regional brain activity during evaluation of emotional stimuli in patients with MDD. Furthermore, these differences are mirrored by DTI changes in the same regions. These DTI changes are in turn explained by the interaction between the allele status of rs1360780 and the experience of maltreatment during childhood. Therefore, our findings provide further evidence that genetic variation in GR function, especially when coupled with a chronically stressful environment in early life, might impact on brain structure in regions involved with emotional processing, thus affecting brain function and possibly leading to an increased vulnerability for MDD.

\section{FUNDING AND DISCLOSURE}

Funding was obtained from the EU through the Marie Curie Programme to Thomas Frodl and Leonardo Tozzi. Funding was obtained from European Union, Initial Training Networks, FP7-PEOPLE-2013-ITN, Project Number: 608346; Project full title: Brain Imaging Return To Health (r'Birth) to TF and LT. The authors declare no conflict of interest.

\section{REFERENCES}

Abe $\mathrm{O}$, Yamasue $\mathrm{H}$, Kasai $\mathrm{K}$, Yamada $\mathrm{H}$, Aoki S, Inoue $\mathrm{H}$ et al (2010). Voxel-based analyses of gray/white matter volume and diffusion tensor data in major depression. Psychiatry Res Neuroimaging 181: 64-70.

Alexander AL, Hurley SA, Samsonov AA, Adluru N, Hosseinbor AP, Mossahebi P et al (2011). Characterization of cerebral white matter properties using quantitative magnetic resonance imaging stains. Brain Connect 1: 423-446.

Arlt S, Demiralay C, Tharun B, Geisel O, Storm N, Eichenlaub M et al (2013). Genetic risk factors for depression in Alzheimer's disease patients. Curr Alzheimer Res 10: 72-81.

Beck AT, Ward CH, Mendelson MM, Mock JJ, Erbaugh JJ (1961). An inventory for measuring depression. Arch Gen Psychiatry 4: 561-571.

Bellivier F, Henry C, Szöke A, Schürhoff F, Nosten-Bertrand M, Feingold J et al (1998). Serotonin transporter gene polymorphisms in patients with unipolar or bipolar depression. Neurosci Lett 255: 143-146.

Bermingham R, Carballedo A, Lisiecka D, Fagan A, Morris D, Fahey C et al (2012). Effect of genetic variant in BICC1 on 
functional and structural brain changes in depression. Neuropsychopharmacology 37: 2855-2862.

Bernstein DP, Fink L (1998). Childhood Trauma Questionnaire: a retrospective self-report manual. The Psychological Corporation: San Antonio, TX.

Binder EB, Bradley RG, Liu W, Epstein MP, Deveau TC, Mercer KB et al (2008). Association of FKBP5 polymorphisms and childhood abuse with risk of posttraumatic stress disorder symptoms in adults. JAMA 299: 1291-1305.

Bosker FJ, Hartman CA, Nolte IM, Prins BP, Terpstra P, Posthuma D et al (2011). Poor replication of candidate genes for major depressive disorder using genome-wide association data. Mol Psychiatry 16: 516-532.

Caspi A, Sugden K, Moffitt TE, Taylor A, Craig IW, Harrington H et al (2003). Influence of life stress on depression: moderation by a polymorphism in the 5-HTT gene. Science 301: 386-389.

Clarke H, Flint J, Attwood AS, Munafò MR (2010). Association of the 5-HTTLPR genotype and unipolar depression: a metaanalysis. Psychol Med 40: 1767-1778.

Cohen-Woods S, Craig IW, McGuffin P (2013). The current state of play on the molecular genetics of depression. Psychol Med 43: 673-687.

Dannlowski U, Ohrmann P, Bauer J, Deckert J, Hohoff C, Kugel H et al (2007). 5-HTTLPR biases amygdala activity in response to masked facial expressions in major depression. Neuropsychopharmacology 33: 418-424.

Dannlowski U, Stuhrmann A, Beutelmann V, Zwanzger P, Lenzen T, Grotegerd D et al (2012). Limbic scars: long-term consequences of childhood maltreatment revealed by functional and structural magnetic resonance imaging. Biol Psychiatry 71: 286-293.

Fani N, Gutman D, Tone EB, Almli L, Mercer KB, Davis J et al (2013). FKBP5 and attention bias for threat: associations with hippocampal function and shape. JAMA Psychiatry 70: 392-400.

Fani N, King TZ, Reiser E, Binder EB, Jovanovic T, Bradley B et al (2014). FKBP5 genotype and structural integrity of the posterior cingulum. Neuropsychopharmacology 39: 1206-1213.

Feldman HM, Yeatman JD, Lee ES, Barde LHF, Gaman-Bean S (2010). Diffusion tensor imaging: a review for pediatric researchers and clinicians. J Dev Behav Pediatr 31: 346-356.

Fitzgerald PB, Laird AR, Maller J, Daskalakis ZJ (2008). A metaanalytic study of changes in brain activation in depression. Hum Brain Mapp 29: 683-695.

Frodl T, Carballedo A, Fagan AJ, Lisiecka D, Ferguson Y, Meaney JF (2012). Effects of early-life adversity on white matter diffusivity changes in patients at risk for major depression. J Psychiatry Neurosci 37: 37-45.

Frodl T, Reinhold E, Koutsouleris N, Donohoe G, Bondy B, Reiser $M$ et al (2010). Childhood stress, serotonin transporter gene and brain structures in major depression. Neuropsychopharmacology 35: 1383-1390.

Gillespie CF, Phifer J, Bradley B, Ressler KJ (2009). Risk and resilience: Genetic and environmental influences on development of the stress response. Depress Anxiety 26: 984-992.

Gizatullin R, Zaboli G, Jönsson EG, Åsberg M, Leopardi R (2006). Haplotype analysis reveals tryptophan hydroxylase (TPH) 1 gene variants associated with major depression. Biol Psychiatry 59: 295-300.

Goeleven E, De Raedt R, Baert S, Koster EHW (2006). Deficient inhibition of emotional information in depression. J Affect Disord 93: 149-157.

Gyekis JP, Yu W, Dong S, Wang H, Qian J, Kota P et al (2013). No association of genetic variants in BDNF with major depression: a meta- and gene-based analysis. Am J Med Genet B Neuropsychiatr Genet 162: 61-70.

Hamilton M (1986)The Hamilton Rating Scale for Depression. In: Sartorius DN, Ban DTA (eds). Assessment of Depression [Internet]. Springer: Berlin Heidelberg [cited 10 November
2014], pp. 143-152. Available from http://link.springer.com/ chapter/10.1007/978-3-642-70486-4_14.

Harvey P-O, Fossati P, Pochon J-B, Levy R, LeBastard G, Lehéricy S et al (2005). Cognitive control and brain resources in major depression: an fMRI study using the n-back task. NeuroImage 26: 860-869.

Holz NE, Buchmann AF, Boecker R, Blomeyer D, Baumeister S, Wolf I et al (2014). Role of FKBP5 in emotion processing: results on amygdala activity, connectivity and volume. Brain Struct Funct 220: $1355-1368$.

Horstmann S, Lucae S, Menke A, Hennings JM, Ising M, Roeske D et al (2010). Polymorphisms in GRIK4, HTR2A, and FKBP5 show interactive effects in predicting remission to antidepressant treatment. Neuropsychopharmacology 35: 727-740.

Ising M, Depping A-M, Siebertz A, Lucae S, Unschuld PG, Kloiber S et al (2008). Polymorphisms in the FKBP5 gene region modulate recovery from psychosocial stress in healthy controls. Eur $J$ Neurosci 28: 389-398.

Kang JI, Chung HC, Jeung H-C, Kim SJ, An SK, Namkoong K (2012). FKBP5 polymorphisms as vulnerability to anxiety and depression in patients with advanced gastric cancer: a controlled and prospective study. Psychoneuroendocrinology 37: $1569-1576$.

Lavebratt C, Aberg E, Sjöholm LK, Forsell Y (2010). Variations in FKBP5 and BDNF genes are suggestively associated with depression in a Swedish population-based cohort. J Affect Disord 125: 249-255.

Lisiecka D, Meisenzahl E, Scheuerecker J, Schoepf V, Whitty P, Chaney A et al (2011). Neural correlates of treatment outcome in major depression. Int J Neuropsychopharmacol 14: 521-534.

Menke A, Klengel T, Rubel J, Brückl T, Pfister H, Lucae S et al (2013). Genetic variation in FKBP5 associated with the extent of stress hormone dysregulation in major depression. Genes Brain Behav 12: 289-296.

Miller AH, Maletic V, Raison CL (2009). Inflammation and its discontents: the role of cytokines in the pathophysiology of major depression. Soc Stress Depress 65: 732-741.

Murphy ML, Frodl T (2011). Meta-analysis of diffusion tensor imaging studies shows altered fractional anisotropy occurring in distinct brain areas in association with depression. Biol Mood Anxiety Disord 1: 3.

Pruessner JC, Dedovic K, Khalili-Mahani N, Engert V, Pruessner M, Buss C et al (2008). Deactivation of the limbic system during acute psychosocial stress: evidence from positron emission tomography and functional magnetic resonance imaging studies. Biol Psychiatry 63: 234-240.

Scharf SH, Liebl C, Binder EB, Schmidt MV, Müller MB (2011). Expression and regulation of the Fkbp5 gene in the adult mouse brain. PLoS One 6: e16883.

Sheline YI, Barch DM, Donnelly JM, Ollinger JM, Snyder AZ, Mintun MA (2001). Increased amygdala response to masked emotional faces in depressed subjects resolves with antidepressant treatment: an fMRI study. Biol Psychiatry 50: 651-658.

Shimony JS, Sheline YI, D’Angelo G, Epstein AA, Benzinger TLS, Mintun MA et al (2009). Diffuse microstructural abnormalities of normal-appearing white matter in late life depression: a diffusion tensor imaging study. Biol Psychiatry 66: 245-252.

Siegle GJ, Thompson W, Carter CS, Steinhauer SR, Thase ME (2007). Increased amygdala and decreased dorsolateral prefrontal BOLD responses in unipolar depression: related and independent features. Biol Psychiatry 61: 198-209.

Sprengelmeyer R, Steele JD, Mwangi B, Kumar P, Christmas D, Milders $\mathrm{M}$ et al (2011). The insular cortex and the neuroanatomy of major depression. J Affect Disord 133: 120-127.

Stuhrmann A, Suslow T, Dannlowski U (2011). Facial emotion processing in major depression: a systematic review of neuroimaging findings. Biol Mood Anxiety Disord 1: 10.

Suslow T, Konrad C, Kugel H, Rumstadt D, Zwitserlood P, Schöning S et al (2010). Automatic mood-congruent amygdala 
responses to masked facial expressions in major depression. Biol Psychiatry 67: 155-160.

Ugwu ID, Amico F, Carballedo A, Fagan AJ, Frodl T (2014). Childhood adversity, depression, age and gender effects on white matter microstructure: a DTI study. Brain Struct Funct $1-13$.

Victor TA, Furey ML, Fromm SJ, Öhman A, Drevets WC (2010). Relationship between amygdala responses to masked faces and mood state and treatment in major depressive disorder. Arch Gen Psychiatry 67: 1128-1138.
White MG, Bogdan R, Fisher PM, Muñoz KE, Williamson DE, Hariri AR (2012). FKBP5 and emotional neglect interact to predict individual differences in amygdala reactivity: FKBP5, emotional neglect and amygdala reactivity. Genes Brain Behav 11: 869-878.

Wochnik GM, Rüegg J, Abel GA, Schmidt U, Holsboer F, Rein T (2005). FK506-binding proteins 51 and 52 differentially regulate dynein interaction and nuclear translocation of the glucocorticoid receptor in mammalian cells. J Biol Chem 280: 4609-4616.

Supplementary Information accompanies the paper on the Neuropsychopharmacology website (http://www.nature.com/npp) 